\title{
A Mouse Embryonic Stem Cell Model of Schwann Cell Differentiation for Studies of the Role of Neurofibromatosis Type 1 in Schwann Cell Development and Tumor Formation
}

\author{
THERESE M. ROTH, ${ }^{1}$ POORNAPRIYA RAMAMURTHY, ${ }^{1,2}$ FUMI EBISU, ${ }^{1,3}$ ROBERT P. LISAK, ${ }^{4}$ \\ BEVERLY M. BEALMEAR, ${ }^{4}$ AND KATE F. BARALD ${ }^{1,2,3 *}$ \\ ${ }^{1}$ Department of Cell and Developmental Biology, University of Michigan Medical School, Ann Arbor, Michigan \\ ${ }^{2}$ The Department of Biomedical Engineering, College of Engineering, University of Michigan, Ann Arbor, Michigan \\ ${ }^{3}$ Program in Neuroscience, University of Michigan, Ann Arbor, Michigan \\ ${ }^{4}$ Department of Neurology, Wayne State University School of Medicine, Detroit, Michigan
}

KEY WORDS

Schwann cells; mouse ES cells; neurofibromin; NF1

\begin{abstract}
The neurofibromatosis Type 1 (NF1) gene functions as a tumor suppressor gene. One known function of neurofibromin, the NF1 protein product, is to accelerate the slow intrinsic GTPase activity of Ras to increase the production of inactive rasGDP, with wide-ranging effects on p21ras pathways. Loss of neurofibromin in the autosomal dominant disorder NF1 is associated with tumors of the peripheral nervous system, particularly neurofibromas, benign lesions in which the major affected cell type is the Schwann cell (SC). NF1 is the most common cancer predisposition syndrome affecting the nervous system. We have developed an in vitro system for differentiating mouse embryonic stem cells (mESC) that are NF1 wild type $(+/+)$, heterozygous $(+/-)$, or null $(-/-)$ into SC-like cells to study the role of NF1 in SC development and tumor formation. These mES-generated SClike cells, regardless of their NF1 status, express SC markers correlated with their stage of maturation, including myelin proteins. They also support and preferentially direct neurite outgrowth from primary neurons. NF1 null and heterozygous SC-like cells proliferate at an accelerated rate compared to NF1 wild type; this growth advantage can be reverted to wild type levels using an inhibitor of MAP kinase kinase (Mek). The mESC of all NF1 types can also be differentiated into neuron-like cells. This novel model system provides an ideal paradigm for studies of the role of NF1 in cell growth and differentiation of the different cell types affected by NF1 in cells with differing levels of neurofibromin that are neither transformed nor malignant. ๑2007 Wiley-Liss, Inc.
\end{abstract}

\section{INTRODUCTION}

NF1 is the most common human cancer predisposition syndrome affecting the nervous system (Gutmann et al., 2001). Hallmarks of NF1 include a range of symptoms from café au lait spots of the skin to tumors of the peripheral nervous system. Schwann cells (SC) have been found to be the originating cell type in most of the benign tumors (Harrisingh and Lloyd, 2004; Zhu et al.,
2002) and can also contribute to malignant peripheral nerve sheath tumors (Lee et al., 2004; Miller et al., 2006). Loss of heterozygosity (LOH) is frequently seen in NF1 tumors, particularly in the SC population (Zhu et al., 2002), although not all SCs in the tumors analyzed showed LOH (Rutkowski et al., 2000; Sawada et al., 1996; Serra et al., 2000).

The functions of neurofibromin, the protein product of the neurofibromatosis Type 1 (NF1) gene, are largely unknown, although investigators have speculated that other activities will be found in addition to its known function of accelerating the hydrolyzation of active rasGTP to inactive rasGDP (Friedman et al., 1999). When NF1 is mutated, Ras is constitutively active, enabling affected cells to proliferate extensively. This proliferation can result in tumor formation, particularly in neural crest-derived cells in the peripheral nervous system, which have the highest neurofibromin expression (Cichowski and Jacks, 2001; Daston et al., 1992; Friedman et al., 1999). A good deal of interest has recently been focused on neural crest-derived SC stem cells and very early stage precursors of SC, which are hypothesized to be the neurofibroma-originating cells in NF1 (Parada et al., 2005; Zhu et al., 2005). It would be very helpful to devise a model system in which differentiation of SC with different levels of neurofibromin could be followed from the "stem cell" to the fully differentiated SC under a variety of environmental conditions, particularly if the cells were as close to "normal" as possiblethat is, neither transformed nor malignant. We have now devised such a model system, using mouse embry-

\footnotetext{
This article contains supplementary material available via the Internet at http:// www.interscience.wiley.com/jpages/0894-1491/suppmat.

Grant sponsor: National Science Foundation; Grant number: 2004016780; Grant sponsor: NIH; Grant numbers: R01 NS17262, DC05939, DC04184; Grant sponsor: AMRMC; Grant number: NF99027 (all to KFB); Grant sponsor: Mary Parker Neuroscience Fund, Detroit Medical Center (RPL).

*Correspondence to: Kate F. Barald, Department of Cell and Developmental Biology, University of Michigan Medical School, 3053 BSRB, 109 Zina Pitcher Place, Ann Arbor, MI 48109-2200, USA. E-mail: kfbarald@umich.edu

Received 10 October 2006; Revised 8 May 2007; Accepted 25 May 2007

DOI 10.1002/glia.20534

Published online 27 June 2007 in Wiley InterScience (www.interscience. wiley.com).
} 


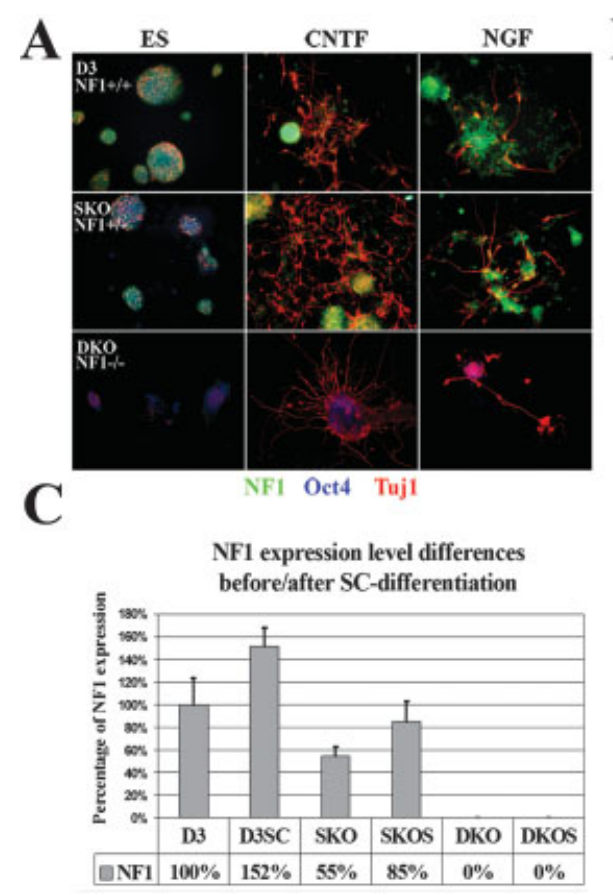

Fig. 1. Undifferentiated mES cells that express various levels of neurofibromin grow in mounds but can be differentiated into neuronlike cells and SC-like cells. NF1 expression is upregulated following SC differentiation. Cell types are listed in the leftmost box of each row. A: Self-renewing mES cells express Oct4 (blue). NF1 expression (green) is strong in D3 $(\mathrm{NF} 1+/+)$ cells, weakly expressed in SKO $(\mathrm{NF} 1+/-)$ cells, and not expressed in DKO (NF1-/-) cells. Tuj1 (red), a neuronal marker, was weakly expressed around the edges of colonies, showing some differentiation in cells on the periphery (left). After 5 days growth in neuronal differentiation media, Tuj1 expression was strongly

onic stem cells engineered to express variable levels of neurofibromin (Jacks et al., 1994). These mouse embryonic stem (mES) cells can be differentiated into most of the cell types affected in NF1.

Previous studies by Sieber-Blum and colleagues demonstrated that neuregulin-1 (Nrg-1) promotes the differentiation of neural crest (NC) stem cells into SCs (Sieber-Blum et al., 2004). Neural crest cells are embryonic transitory migratory cells that differentiate into many cell types (Dorsky et al., 2000; Kalcheim and Burstyn-Cohen, 2005; Le Douarin et al., 2004; Lobsiger et al., 2002), including those of the peripheral nervous system affected in NF1, including SCs and neurons (Cichowski and Jacks, 2001; Daston and Ratner, 1992; Vogel et al., 1995). Before becoming mature SC, which can be either myelinating or nonmyelinating, NC cells pass through SC precursor and immature SC stages. Myelination by SC requires the presence of large diameter neurites (Bunge et al., 1980, 1982; Ratner et al., 1986).

We have used media containing neuregulin to produce a SC differentiation model using mouse ES (mES) cells that have one $(\mathrm{NF} 1+/-)$, both $(\mathrm{NF} 1+/+)$, or neither (NF1-/-) functional allele. All three cell types have the potential to differentiate into many different kinds of cells, including neuron-like cells (Fig. 1A) and Schwannlike cells (Fig. 1B). They provide an ideal system to study the effects of the environment on the gene expres-

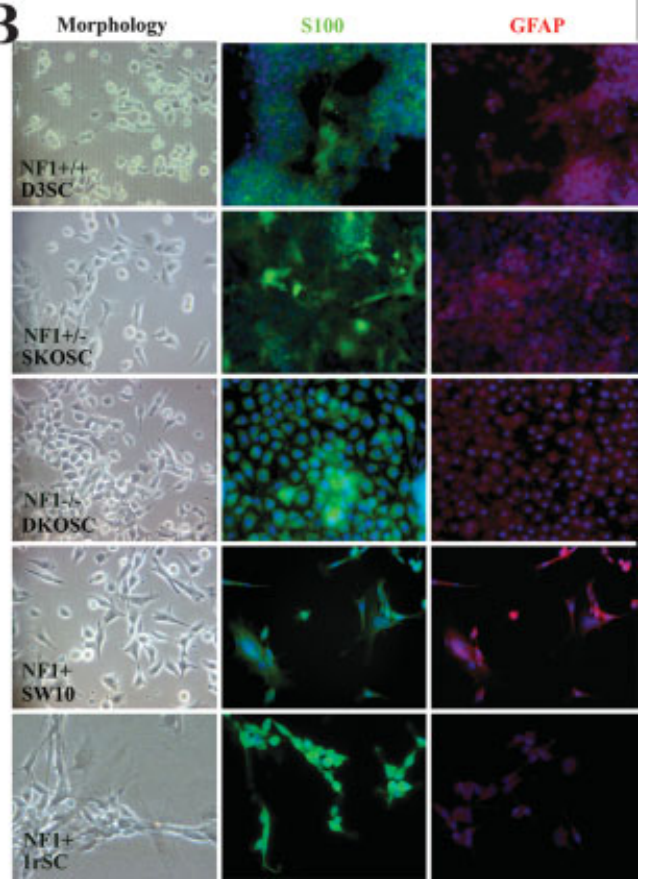

observed in the neurites of neuron-like cells; Oct4 expression decreased and was only seen in some clumps of cells in large colonies (center and right). B: Left column illustrates SC-like morphology of mES cells, similar to the morphology of the SW10 SC line and primary rat SC. $(\times 32$ mag. with phase 1 filter). Middle column shows expression of S100 and right column shows GFAP expression $(\times 40 \mathrm{mag})$. C: RTqPCR shows NF1 expression in SKO cells is 55\% of the level in D3 cells; expression in DKO cells is undetectable. After SC differentiation, NF1 expression increased $\sim 150 \%$ in both D3SC cells and SKOSC cells, but is undetectable in DKOSC cells.

sion cascades involved in the differentiation of cell types that make variable levels of neurofibromin and that are implicated in the progression of neurofibromatosis 1 .

\section{MATERIALS AND METHODS Cell Types}

The cell lines used for these studies include the following: the D3 $(\mathrm{NF} 1+/+)$ mES cell line (Doetschmann, 1985), the SKO (NF1+/-) mES cell line (Jacks et al., 1994), and a DKO (NF1-/-) mES cell line with the NF1 gene disrupted by insertion of a neo cassette replacing exon 31 (Jacks et al., 1994). Control cell lines included the SW10 (NF1+/+) mouse SC line, which carries a temperature sensitive SV40 large $\mathrm{T}$ antigen and which is grown at $37^{\circ} \mathrm{C}$ (the nonpermissive temperature for transgene expression) for differentiation studies (ATCC, Manassas, VA), as well as primary rat SC, grown as previously described (Lisak and Bealmear, 1991).

\section{Media}

Proliferating (ES): $81 \%$ DMEM without phenol red, $1 \%$ L-Glut, $1 \%$ Pen/Strep, $1 \%$ nonessential amino acids (Gibco, Carlsbad, CA), 15\% FBS (Atlanta Biological, Nor- 
cross, GA), $1 \%$ sodium pyruvate ( $2 \%$ stock), $7 \mu \mathrm{L} / \mathrm{L} \beta$ mercaptoethanol (Sigma, St. Louis, MO), 1,000 U/mL ESGRO (Chemicon, Temecula, CA). Neuronal differentiation: 95\%F$12,1 \%$ Pen/Strep, $1 \%$ N2, $2 \%$ B27 (Gibco), $2 \%$ sodium pyruvate (2\% stock), $0.5 \mu \mathrm{g} / \mathrm{L}$ bFGF, $1 \mu \mathrm{g} / \mathrm{L}$ IL-7, $5 \mu \mathrm{g} / \mathrm{L}$ IGF-1 (Sigma), either $1 \mu \mathrm{g} / \mathrm{L}$ CNTF $(\mathrm{R}+\mathrm{D})$ or $1 \mu \mathrm{g} / \mathrm{L}$ NGF (Chemicon). SC differentiation: $84 \% \alpha$-modified MEM without phenol red, 1\% Pen/Strep (Gibco), 10\% FBS (Atlanta Biological), 5\% 11-day chick embryo extract, $10 \mathrm{ng} / \mathrm{mL}$ NRG-1 (R\&D systems, Minneapolis, MN). SW10 media: 88\% DMEM without phenol red, 1\% l-glutamine, 1\% Pen/Strep, $10 \%$ FBS (Atlanta Biological). Primary rat SC media: 90\% DMEM (Gibco), 10\% FBS (Atlanta Biological). Minimal media: 87\% DMEM without phenol red, 1\% Pen/Strep, 1\% lglutamine (Gibco), 10\% FBS (Atlanta Biological), 1\% sodium pyruvate (Gibco).

\section{Antibodies}

We used antibodies to: NF1 (rabbit polyclonal 1:50), Oct4 (goat polyclonal 1:50) (Santa Cruz, CA), Tuj1 (mouse monoclonal 1:500) (Covance, Berkeley, CA), Neurofilament (rabbit polyclonal 1:400) (Chemicon, Temecula, CA), S100 (rabbit polyclonal 1:200) (Novocastra, Newcastle Upon Tyne, UK) or mouse monoclonal (1:50) (Abcam, Cambridge, MA), GFAP (mouse monoclonal 1:400) (Chemicon), peripheral myelin basic protein (mouse monoclonal 1:10), Ki67 (rabbit polyclonal 1:100) (Abcam), $\alpha$-tubulin (mouse monoclonal 1:200), AlexaFluor 350, 488, 594, DAPI (Molecular Probes, Eugene, OR), goat $\alpha$ rabbitHRP (1:500) (Zymed, San Francisco, CA).

\section{Reagents}

Reagents included: Histomouse kit (Zymed), Prolong Gold anti-fade (Molecular Probes), Porcine gel (Sigma), CellTiter96 cell proliferation assay (Promega). All antibodies were diluted in $10 \%$ donkey serum (Chemicon) in $0.1 \%$ Tween20/phosphate buffered saline (PBS) (Sigma).

\section{Differentiation of mES Cells into Neuron-Like Cells}

mES cells in ES medium were plated onto Primaria (Falcon) plates and allowed to attach overnight. After $24 \mathrm{~h}$, cells were washed $1 \times$ with PBS (GIBCO formulation) and the medium was changed to neuronal differentiation medium containing either $1 \mu \mathrm{g} / \mathrm{L}$ CNTF or $1 \mu \mathrm{g} / \mathrm{L} \mathrm{NGF}$. Undifferentiated mES cells were fed daily, while cells in neuronal differentiation media were fed every other day. After 5 days' growth, cells were labeled for expression of NF1, Oct4 (selfrenewing mES cells), and Tuj1 (neuronal differentiation).

\section{Differentiation of mES Cells into SC-Like Cells}

mES cells in ES medium were plated onto Primaria (Falcon) plates and allowed to attach overnight. After
$24 \mathrm{~h}$, cells were washed $1 \times$ with $\mathrm{PBS}$ and the medium was changed to SC medium. Cells were fed every other day and passed when confluent ( every 5-6 days). After 1 week, cells had the appearance of SC-like cells (flat and spindly), and proliferation rate was assessed and found to have decreased. After 2 weeks in SC-differentiation medium cells were examined by RTqPCR for changes in NF1 gene and protein expression and immunoassayed for S100 and GFAP expression. After 1 month, cells were assayed for the ability to initiate and direct neurite outgrowth and to produce myelin. Cells have been frozen and thawed and remain proliferative and SC marker-expressing over at least 20 passages.

\section{Immunocytochemistry}

About 40,000 cells were plated onto $0.1 \%$ porcine gelcoated (Sigma) coverslips (Corning, Corning, NY) and grown overnight. Cells were then fixed in $4 \%$ paraformaldehyde (Sigma), permeabilized in $0.2 \%$ TritonX-100 (Sigma), stained, and mounted onto Superfrost plus slides (Fisher, Pittsburgh, PA). The Zymed Histomouse kit was used according to the manufacturer's instructions for nonfluorescent ICC using mouse monoclonal antibodies. Fluorescence micrographs were taken with an Olympus BX-51 microscope, and nonfluorescent micrographs with Nikon ACT-1 software on a Leitz Diavert inverted microscope.

\section{RTqPCR}

RTqPCR was performed using primer pairs designed using the Beacon designer program (BioRad, Hercules, $\mathrm{CA}$ ), with target $\mathrm{Ta}$ at $55^{\circ} \mathrm{C}$, length $18-22 \mathrm{bp}$, and amplicon size 100-200. SW10 gene expression was normalized to GAPDH. A comparison of gene expression in SC-like mES cells with undifferentiated mES cells was done by dividing the expression in SC differentiated cells by the expression in proliferative undifferentiated mES cells. Quantification of SC marker expression was assayed at the time points listed in supplemental Table 1. To assay SC-like cells' myelin gene upregulation in the presence of neurons, day E5 chick statoacoustic ganglia (SAGs) were excised and added at time points listed and grown an additional 7 days before RTqPCR analysis. Total RNA from the samples was extracted using Qiagen mRNA Kit 74106 (Qiagen, Valencia, CA).

RT-PCR was performed as follows: cDNA was synthesized from $2 \mu \mathrm{g}$ total RNA by reverse transcription using Super Script III transcriptase (Invitrogen, Carlsbad, CA) and oligo dT primer. The PCR conditions included an initial denaturation at $94^{\circ} \mathrm{C}$ for $1 \mathrm{~min}, 94^{\circ} \mathrm{C}$ for $1 \mathrm{~min}$, $55^{\circ} \mathrm{C}$ for $30 \mathrm{~s}$ and $74^{\circ} \mathrm{C}$ for $30 \mathrm{~s}$ and 34 cycles and final extension at $72^{\circ} \mathrm{C}$ for $5 \mathrm{~min}$ and $4^{\circ} \mathrm{C}$ holding temperature. The PCR products were separated on $2.0 \%$ agarose gels and visualized using ethidium bromide (staining) under UV light. 


\section{PCR Primers}

NF1: forward: AGTTTCTCTCCTCGCTGGTCTTC; reverse: CGTTTCCTGCCACCCGTTTG; Oct4: forward: CCAACGA GAAGAGTATGAGG; reverse: CTGAGTAGAGTGTGGTGA AG. a4integrin: forward: GAATCTCCTCCACCTACTCAC AG; reverse: CCAACGGCTACATCAACATATCC; Cad19: forward: GGAGGAGACAGACAAGATG; reverse: TGAAGGA GTGATGGTAGATG; PMP22: forward: GCTCTGTTCCTG TTCTTC TG; reverse: TGCCTCACTGTGTAGATGG; MBP: forward: AGACCCTCACAGCGATCCAAG; reverse: AGTC AAGGATGCCCGTGTCTC; $\alpha 1 \beta 1$ integrin: forward: CAGTG AATGGCAACAATGAAG; reverse: ATCAGCAGCAAGGC AAGG.

Measurement of Proliferation rates of mES cells during SC-like differentiation: 30,000 cells per well were counted and plated onto Primaria (Falcon) 24-well plates. Proliferation was assayed at specific time points using immunocytochemistry with the Ki67 antibody, which recognizes cells in active cell cycle. If indicated, E5 chick statoacoustic ganglia (SAGs), which are destined to innervate the inner ear, were excised and added to the plate and grown for an additional 3 days before the assay was performed. The percentage of Ki67-positive cells compared to total cell number was used to determine the proliferation rate. Microsoft Excel was used to graph and analyze the data, which are expressed as mean \pm SD.

\section{Proliferation Rates of mES Cells After Addition of U0126 Mek Inhibitor}

To determine whether the Ras pathway might be implicated in overproliferation of $\mathrm{NF} 1+/-$ and $\mathrm{NF} 1-/-$ SC-like cells compared to wild type cells, 10,000 cells per well were plated onto 96-well Primaria plates. After $1 \mathrm{~h}$, either $4 \mu \mathrm{M}$ U0126 (Mek inhibitor) or ethanol (vehicle control) was added to wells and cells were allowed to grow for 3 days. Proliferating cells were identified using the CellTiter96 (Promega) proliferation assay and absorbance was measured at $492 \mathrm{~nm}$ by a microplate reader (Fisher). Absorbance is directly proportional to the number of actively cycling cells.

\section{Statoacoustic Ganglion Neurite Outgrowth and Directional Outgrowth in the Presence of SC-Like Cells}

To test for SC-supported neurite outgrowth and directed neurite outgrowth, statoacoustic ganglia (SAGs) from E5 chick embryos (Bianchi et al., 2005) were excised and plated onto undifferentiated mES cells (D3, $\mathrm{SKO}$, or DKO), SC-differentiated mES cells (D3SC, SKOSC, DKOSC), or SW10SC cells. After 5 days, cultures were assayed for the presence or absence of neurite outgrowth (neurofilament positive neurites) as well as the percentage of the area occupied by SAG-neurite positive cells and processes. The total area of SAG neurite positive cells and process outgrowth was quantified using Metamorph software (Universal Images Corp) threshold image analysis by determining the percent of Neurofilament-stained cell/neurite area compared to the total area.

To determine whether SAG neurite outgrowth was preferentially directed towards SC-differentiated cells, we excised and plated SAGs on culture dishes to which undifferentiated mES cells and SC-like cells were subsequently added on coverslips in two different quadrants of the plate separated by $180^{\circ}$ but equidistant from the SAG at the center. We first allowed the SAGs to attach overnight and then added the two coverslips; one containing undifferentiated mES cells and the other coverslip containing SC-like cells. These cultures were grown for 5 days and assessed for (a) preferential direction of neurite outgrowth, (b) any contact between the mES cells (SC-differentiated or undifferentiated) and the neurites. Neurites in each half of the plate were assessed for extension toward each cell type (undifferentiated vs. SC-differentiated).

\section{RESULTS \\ SC-Like Morphology in Neuregulin-Containing SC Medium}

SC lineage markers characteristic of maturation are sequentially expressed and NF1 expression increases in cultures of mES-derived SC-like cells.

We exposed the (D3 NF1+/+, SKO NF1+/-, and DKO NF1-/-) mES cells to SC differentiation medium. After growing for 1 week in SC media, fed every second day, all cell types (D3 NF1+/+, SKO NF1+/-, and DKO NF1-/-) flattened out and took on the spindly appearance characteristic of SC. All these cells also expressed two different SC proteins, S100 and GFAP, as did the SW10 mouse SC line and the primary rat SC (Fig. 1B, compare with mouse SC line SW10 and primary rat SC in bottom rows). Hereafter, SC-like differentiated $\mathrm{mES}$ cells will be referred to as D3SC, SKOSC, and DKOSC, for D3 (WT-NF1+/+), SKO (single knock out-that is missing one NF1 allele; NF1+/-), or DKO (double knock out; NF1-/-) respectively.

Because exon 31 had been replaced by a neo cassette in these mutant NF1 cells (Jacks et al., 1994), we were able to target this area to design primers to quantify NF1 expression. RTqPCR confirmed NF1 expression in undifferentiated D3 and SKO cells. NF1 was not expressed in DKO cells (Fig. 1C). The NF1 expression levels in D3 cells were set at $100 \%$. This level was used to determine NF1 expression levels of SKO (55\%) and DKO $(0 \%)$ cells, and all SC-differentiated cells. NF1 expression was upregulated $\sim 150 \%$ after 2 weeks in SC-differentiation media in D3SC (from 100 to $152 \%$ ) and SKOSC (from 55 to 85\%) cells, while remaining at undetectable levels in DKOSC (Fig. 1C). NF1 was also expressed in SW10 SC and primary rat SC not shown).

To determine whether all three differentiating mES cell types followed all the characteristic stages of differ- 

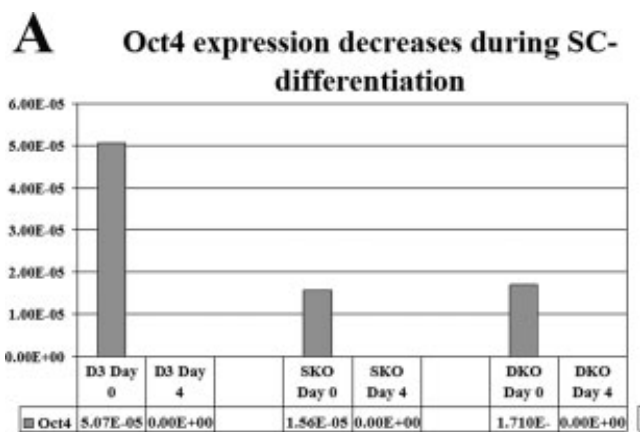

C
Cad19 expression increases during SC-differentiation

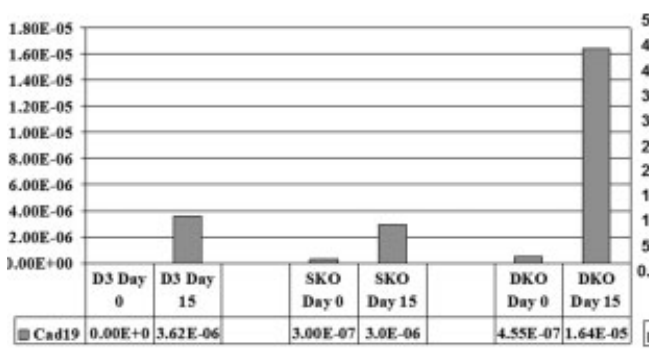

$\mathbf{E}$

SAG effect on MBP expression increase during $\mathrm{SC}$-differentiation

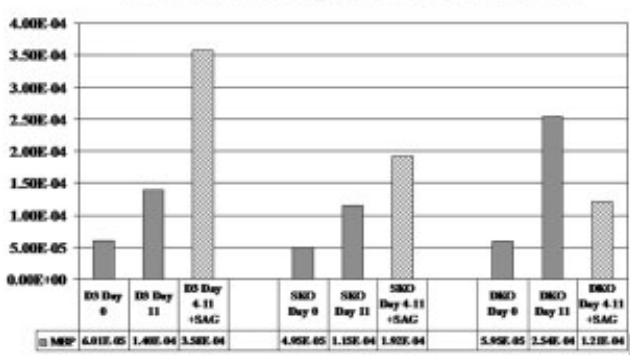

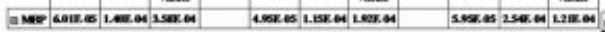

B a4integrin expression increases during SC-differentiation

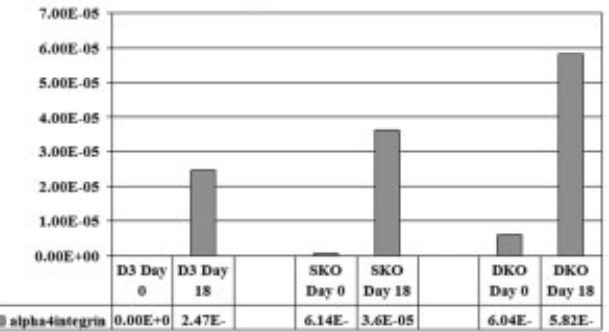

D
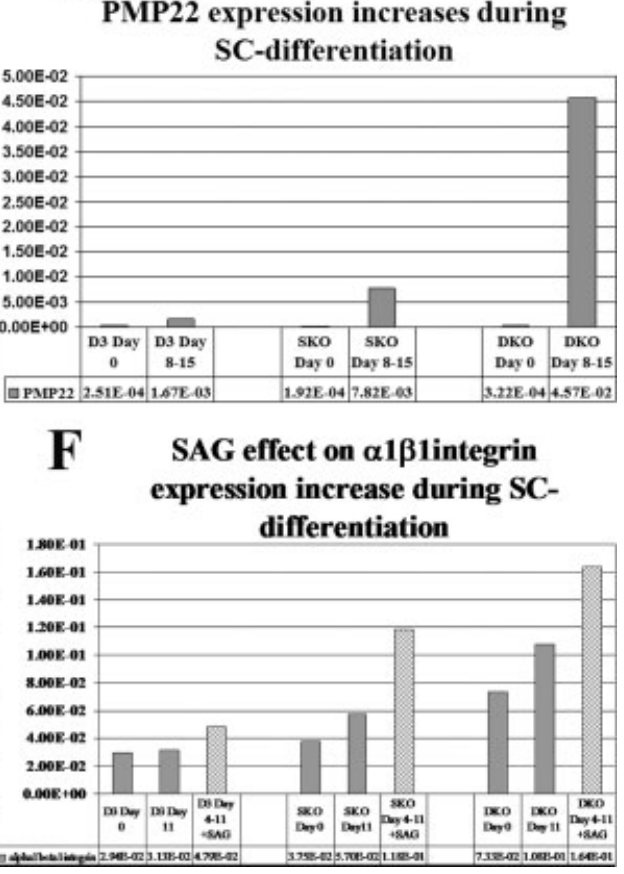

tectable or expressed at very low levels in undifferentiated ES cells but are all detectable following culture in SC differentiation medium. $\mathbf{E}$ and F: Two markers of mature SC are upregulated in the presence of SAG neurites. MBP, expressed in myelinating SC, is more highly upregulated in D3SC $(\mathrm{NF} 1+/+)$ cells; $\alpha 1 \beta 1$ integrin, expressed in nonmyelinating SC, is most highly expressed in DKOSC (NF1-/-) cells. entiation into mature $\mathrm{SC}$, we also examined gene expression at various stages of differentiation from undifferentiated ES cells to mature SC, using as markers genes characteristic for these stages (Jessen and Mirsky, 1991; Lobsiger et al., 2002). These included the following stages/markers: Undifferentiated ES (Oct4), neural crest stem cell ( $\alpha$ 4integrin), SC progenitor (Cad19), immature SC (PMP22), to mature myelinating SC (myelin basic protein-MBP) or nonmyelinating SC ( $\alpha 1 \beta 1$ integrin). We found that Oct4 (Fig. 2A) was most highly expressed on day 0 in culture, becoming undetectable in all cell types with continued culture in SC-differentiation media. Both DKO and SKO cells expressed lower levels of Oct4 on day 0 than D3 cells. By contrast, markers of SC-differentiation, such as $\alpha 4$ integrin (Fig. 2B) and Cad19 (Fig. 2C), were more gradually upregulated, while genes characteristic of more mature SCs, including PMP22
(Fig. 2D) the expression of which persists through myelination, MBP (Fig. 2E), and $\alpha 1 \beta 1$ integrin (Fig. 2F), generally needed the presence of SAG neurites to stimulate their highest expression levels (Fig. 2 and supplementary Table 1). An exception to this was seen in DKOSC (NF1-/-) cells, in which MBP was downregulated in the presence of SAG neurites.

\section{Effect of The Overproliferation of NF1-Deficient or Null SC-Like Cells Can Be Reduced by Exposure to a Mek Inhibitor, U0126, on Proliferation Rates}

$\mathrm{NF} 1+/+$ mES cells had higher proliferation rates than NF1 deficient mES cells (Fig. 3A, day 0), but after 

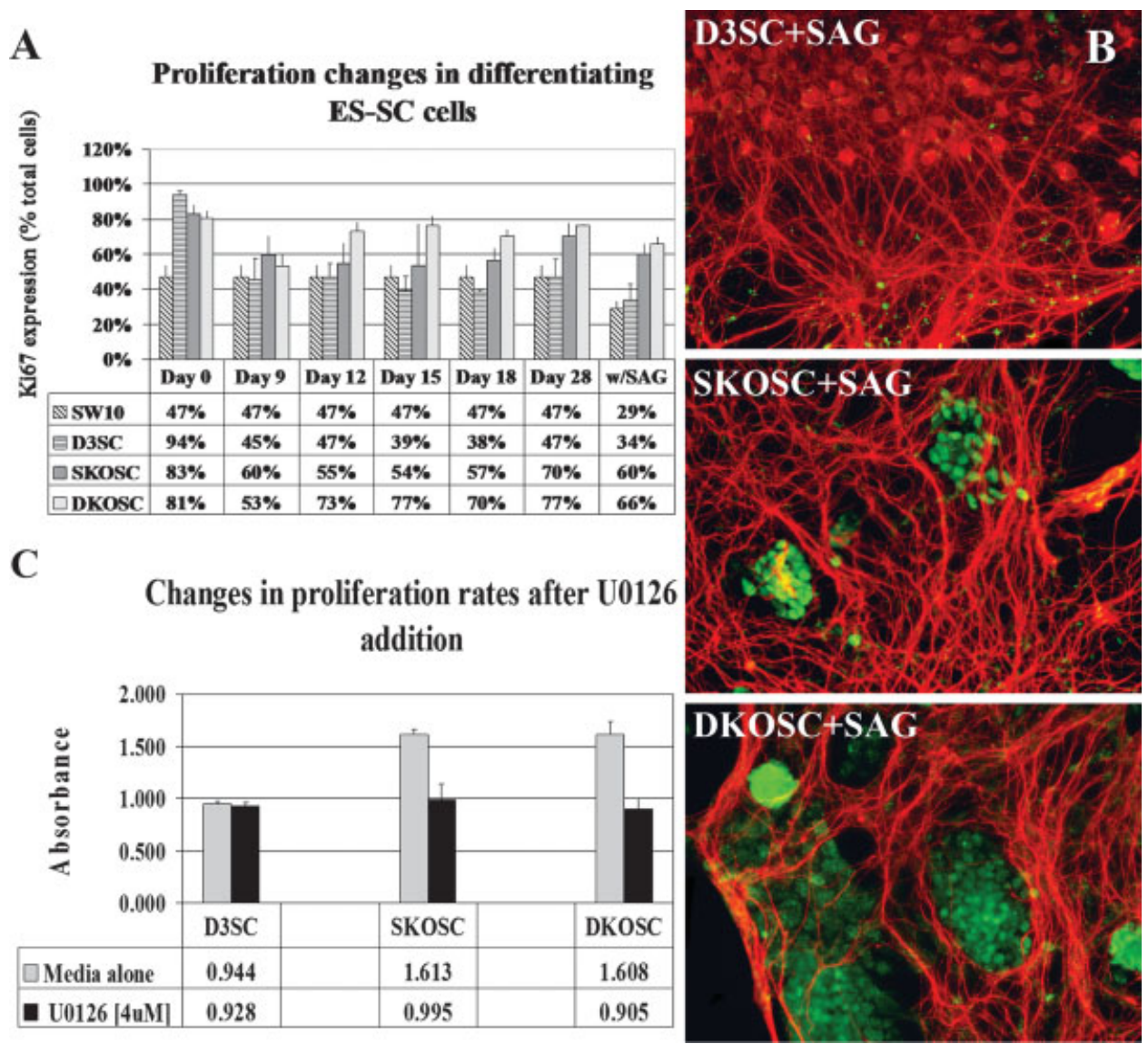

Fig. 3. Proliferation rates differ among cell types based on NF1 genotype. A: NF1+/+ mES cells have higher proliferation rates, determined by percentage of cells in active cell cycle (Ki67+) than NF1 deficient mES cells (day 0). SC-like differentiated NF1 deficient cells had higher proliferation rates than $\mathrm{NF} 1+/+$ cells. By 28 days in SC-differentiation media, D3SC cells had the same percentage $(47 \%)$ of cells in cell cycle as SW10 SC, while SKOSC and DKOSC cells were hyperproliferative (70 and 77\%, respectively). Following addition of SAG cells to the culture, NF1+/+ cells (D3SC, SW10) showed reduced proliferation, with only about $30 \%$ of cells in cell cycle, while NF1+/- (SKOSC) and NF1-/- (DKOSC) still had over $60 \%$ of cells in cell cycle. B: Staining for Ki67-positive cells: proliferative clumps of nonaxon associated SKOSC and DKOSC cells are seen (green) (×100 mag.). C: Overproliferation of NF1+/- and NF1-/- SC-differentiated cells may be dependent on Ras signaling. SKOSC (NF1+/-) and DKOSC (NF1-/-) cells proliferate at a significantly increased rate over that of D3SC $(\mathrm{NF} 1+/+)$ cells. This overproliferation can be decreased to wild type levels through application of $4 \mu \mathrm{M}$ U0126, an inhibitor of the Mek component of the Ras pathway.

day 9 in SC-differentiating conditions NF1 deficient cells had higher proliferation rates than $\mathrm{NF} 1+/+$ cells (D3SC) (Fig. 3A, days 18 and 28), which could be due to constitutively active Ras. D3SC cells' proliferation rate was comparable to that of SW10 SC (47\%). As quiescence is expected for SC in the presence of neurites, following chick statoacoustic ganglion (SAG) addition to the culture, NF1+/+ cells (D3SC, SW10) became less proliferative, with 34 and 29\% actively cycling cells, respectively (Fig. 3A, rightmost set). By contrast, most SKOSC (70-60\%) and DKOSC (77-66\%) cells remained in active cell cycle (Fig. 3A). Ki67 expression was seen in nonaxonal-associated clumps of proliferative SKOSC $(\mathrm{NF} 1+/-)$ and DKOSC $(\mathrm{NF} 1-/-)$ cells among the SAG neurites (Fig. 3B).

The overproliferation seen in SKOSC and DKOSC cells could be reverted to WT levels by the addition of $4 \mu \mathrm{M}$ U0126, a Mek inhibitor (Favata et al., 1998), implicating the Ras pathway in this overproliferation, since this pathway is constitutively active following the loss of NF1 function (Fig. 3C).

\section{Effect of SC-like Differentiation of mES Cells on Myelin Basic Protein Expression and Neurite Outgrown from Statoacoustic Ganglia}

We found that neurite outgrowth and complexity of neurite formation and branching in chick statoacoustic ganglia (SAG) was increased in the presence of $\mathrm{mES}$ cells that had become SC-like (Fig. 4). Excised SAGs from E5 chick embryos plated onto undifferentiated $\mathrm{mES}$ (D3, SKO or DKO) cells, SC-differentiated (D3SC, SKOSC, or DKOSC) mES cells or SW10 SC cells had markedly different neurite outgrowth patterns. We found that undifferentiated mES cells, regardless of their NF1 genotype and NF1 expression level, did not support either neurite outgrowth or myelin production, while SAGs grown on any of the SC-differentiated cells (Fig. 4A) supported extensive neurite outgrowth over 5 days, with branching processes that were never seen in cultures of SAGs alone (Fig. 5B), or on defined substrates such as poly-L-lysine, even in neurite outgrowth promoting medium (Bianchi et al., 2005). Sometimes 
Fig. 4. mES cells which had been differentiated into SC-like cells, but not undifferentiated mES cells, directionally stimulated neurite outgrowth from chick SAGs. Cell types are listed in each box. A: E5 chick SAGs were labeled with antibodies to Tuj1 (red). Little or no neurite outgrowth was directed toward undifferentiated mES cells, but extensive outgrowth was seen toward SC-like cells (Undifferentiated photographs $\times 20$ and SC-like cells $\times 10$ mag.). B: SAGs plated on the TC substrate equidistant between coverslips of undifferentiated mES cells (left side) and SC-like differentiated mES cells (right side): Neurite outgrowth was preferentially directed towards the SC-like cells. Undifferentiated mES cells are seen as purple mounds ( $\times 4$ mag.).
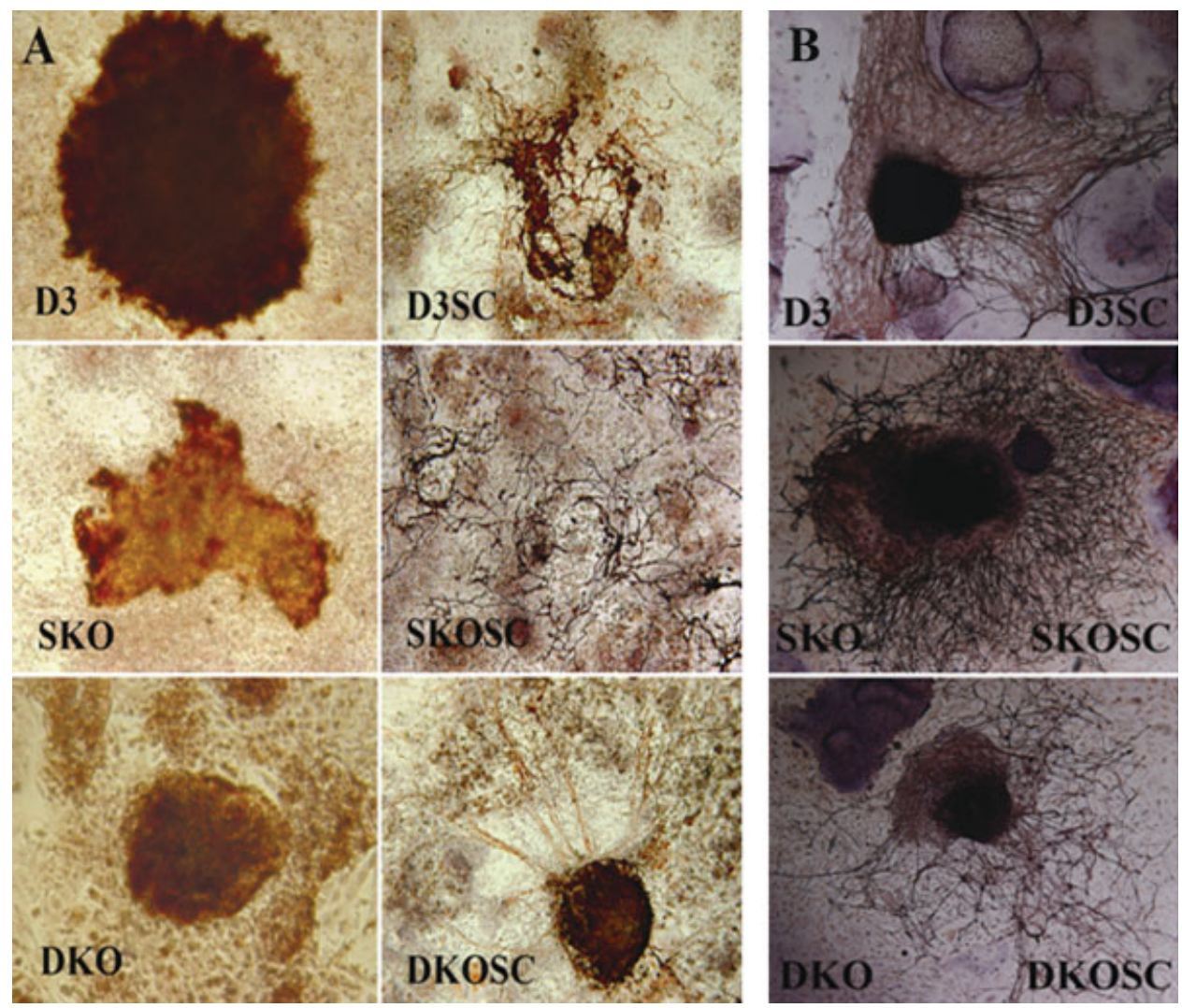

outgrowth was so extensive, especially on NF1 deficient and NF1 null cells, that it obscured our ability to discern the shape of the original SAG (especially see SKOSC Fig. 4A, middle row to the right).

We next determined whether the SAGs needed physical cell-cell contact with the SC-like cells or if the cells could affect the directionality of neurite outgrowth without direct contact. Excised SAGs were plated on culture dishes on which undifferentiated mES cells (Fig. 4C, on the left) and SC-like cells (Fig. 4C, on the right) were subsequently added equidistant from the SAG center on coverslips in two different quadrants of the plate $180^{\circ}$ apart. After 5 days growth, in every case the SAGs preferentially extended neurites toward/over the SC-like $\mathrm{mES}$ cells, and almost invariably veered around undifferentiated ES colonies without making contact (Fig. 4C). Although undifferentiated ES cells did not seem to inhibit the initiation of neurite outgrowth, neurites that had begun to grow out from the side of the explant nearer the undifferentiated ES cells turned and extended towards the differentiated SC-like mES cells ( $n=4$ for each cell type). Over the 5-day observation period, the cells of both types continued to proliferate (ES cells at a faster rate than the SC-like cells) (Fig. 4C), with cells migrating off the coverslip, so that the undifferentiated cells sometimes grew very near to the SAGs, causing the neurites to veer around these colonies (purplish mounds) of undifferentiated mES cells (Fig. 4). When neurite outgrowth in the half of the plate that contained SC-differentiated mES cells was compared to that in the other half of the plate, occupied by undifferentiated mES cells, the direction of neurite outgrowth was always found to be towards the SC-like cells; neurites that began by traveling toward the undifferentiated cells changed course and veered towards the SClike cells in every case $(n=5)$. Even though the cells were grown without LIF (ES) or Nrg-1 (SC) supplements to the medium, they kept their respective morphological appearance over the 5 days of the assay.

\section{Expression of Myelin Basic Protein, SAG Neurite Extension and Branching}

To assay whether the SC-like cells were recapitulating the maturation patterns of rodent primary SC that are normally seen in vitro as well as in vivo studies (Bunge et al., 1980), we determined whether the SC-like mES cells expressed myelin proteins and became associated with neurites in the presence of primary SAG neurons. We plated and cultured the SAGs and either SC-like cells or SW10 SC for 5 days, after which the cells were labeled for either PNS myelin protein expression using a mouse monoclonal antibody to peripheral myelin and the Histomouse kit (Fig. 5A, left column), or analyzed for a combination of Tuj1 (for neurites) and DAPI (nuclear stain) for visualizing SCs' association with neurites (Fig. 5A, right column). With the Histomouse kit, we were able to demonstrate that the SC-like cells as well as SW10 SC that did express myelin clustered around 
A

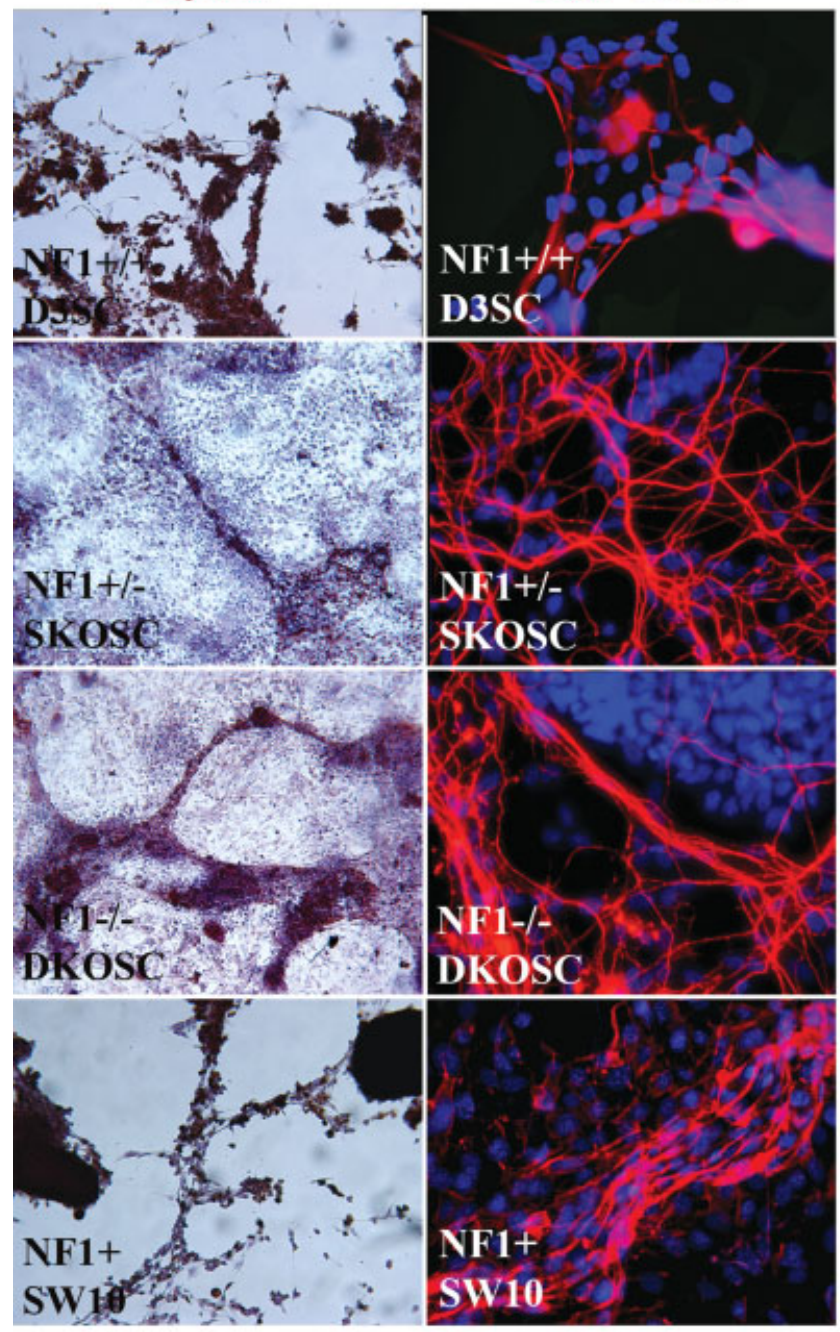

B
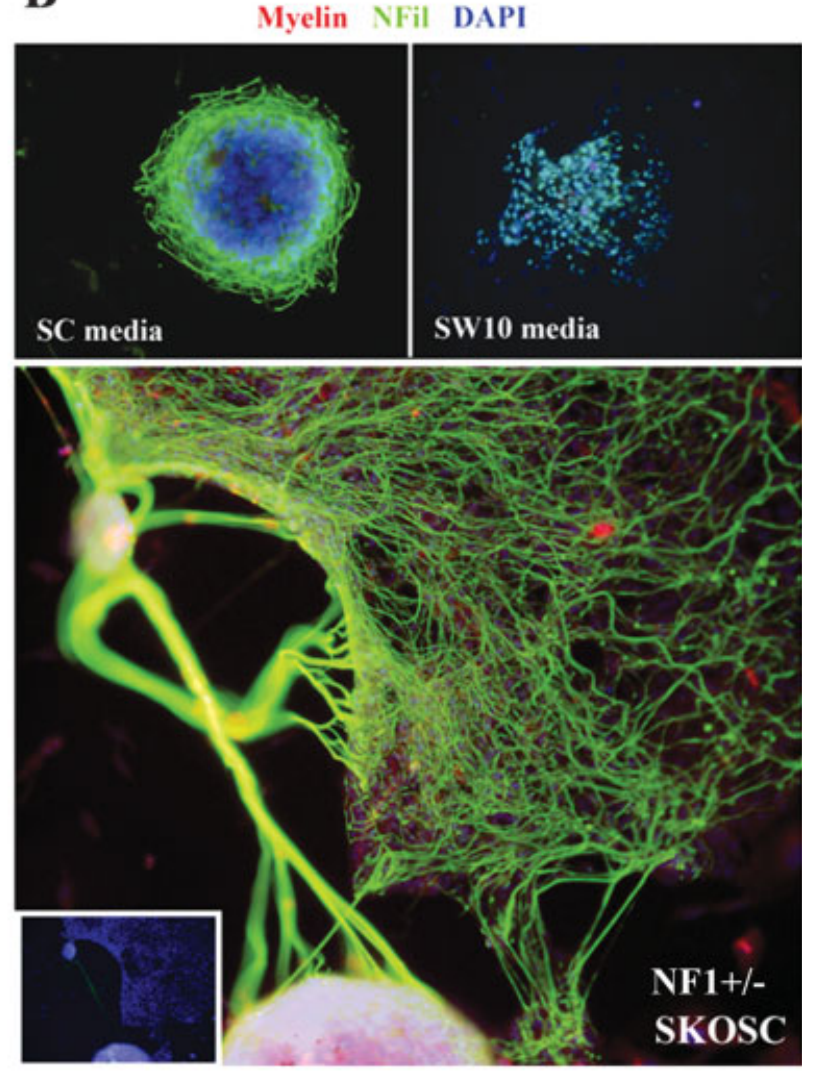

C
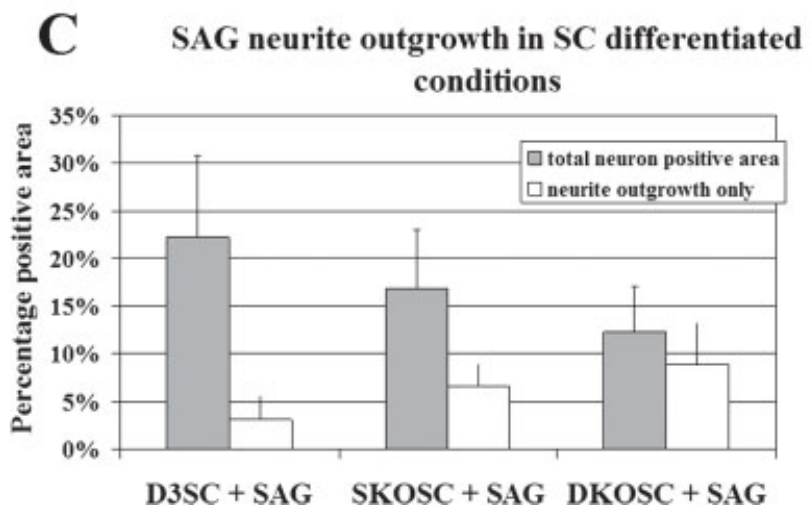

grown in media alone without an underlying cell layer did not extend neurites or express myelin $(\times 40$ magnification $)$. C: SAG neurite outgrowth is dependent on NF1 status of underlying cells. Greater migration and neurite outgrowth and branching of the SAG neurites is seen in the presence of SKOSC (NF1+/-) (lower panel in B) and DKOSC (NF1-/-) cells. C: Neurite extensions and neuronal cell somata (Tuj1 stained regions) were measured using Metamorph threshold image analysis software. Although total neuron/neurite area was greatest in cultures of D3SC, neurite area was greatest in DKOSC cultures. what appear to be axons that were expressing neuronal markers (Fig. 5A, left column). In the two NF1+/+ cell lines (D3 and SW10), myelin positive cells were associated with the axons of the SAG and there was very little SC growth anywhere on the rest of the plate. In SKOSC and DKOSC cultures, however, cells farther away from the neurites did not express myelin and became very confluent. Many clusters of SKOSC and DKOSC were seen not to be associated with neurites (Fig. 5A, left and right column).

To be sure that cells that may have been excised with the SAG were not those producing the observed myelin expression, we also grew SAGs on plates without any additional cells in the same medium used to culture SC or SW10 cells. SAGs or associated cells did not express myelin under any of these conditions (Fig. 5B top two 
boxes). Most SAGs did not extend any neurites at all under these conditions (Fig. 5B, top right); occasionally, neurite extension was initiated, but these neuritic processes were confined to areas around the SAG, without extending onto the plate (Fig. 5B, top left).

Next, we wanted to determine whether there were differences in the area of the plate occupied by neurons or neurites that varied with the levels of NF1 expression in the co-cultured SC. To quantify the amount of neurite outgrowth, we used the Metamorph software program to measure the percentage of the area covered by neurite outgrowth, as represented by Tuj1 or neurofilament antibody staining, compared to the total area. We found that D3SC cells stimulated SAGs and their associated neurites to cover significantly more area than SKOSC cells or DKOSC cells (Fig. 5C). Although the D3SC cells stimulated more total neuron/neurite-positive area, much of this area was seen to be in clumps of the SAG cell bodies themselves rather than in neurite extensions over the SC lawns. SAGs grown in the presence of SKOSC and DKOSC cells appeared to have more neurite branching over the areas of the plate with underlying SC-like cells (Fig. 5B, bottom). We next subtracted the Tuj1- or neurofilament-positive clumps of SAG somata from the total area and measured only the extent of neurite outgrowth and branching seen with the Metamorph program. DKOSC and SKOSC cultures had higher percentages of neurite positive areas compared to the total area than D3SC (Fig. 5C). However, SKOSC and DKOSC also had more SC-like mES proliferating cells that were not associated with SAG neurites (Fig. 5A) than were found in co-cultures with D3SC.

\section{DISCUSSION}

We have devised a model system to study the role of NF1 in SC development and tumorigenesis in which the levels of neurofibromin are known prior to differentiation and the developmental stage/status in their progression along the SC maturation pathway is also known. More importantly, they are neither transformed nor malignant, as are most of the human or rodent cell lines used in studies of NF1, especially those used in tumor progression studies. Comparisons of the role of NF1 in the stem cells' differentiation into neurons, SC, and other cell types affected in the disorder should allow us to identify key steps in the differentiation processes that may be susceptible to malignant transformation. In this study, mES cells were differentiated into SC-like cells in order to more closely approximate the originating cell type in both neurofibromas and more advanced tumors (Zhu et al., 2002), which is now believed to be a stem cell or early SC progenitors rather than the mature differentiated SC.

Neuregulin-1 (Nrg-1) had been found to differentiate neural crest stem cells into SCs (Sieber-Blum et al., 2004). We used a modified version of this Nrg-1-containing medium to direct the differentiation of wild type (D3) mES cells into SC-like cells, which in their mor- phology, reduction of proliferation rates and sequential acquisition of SC markers as they matured in culture, closely resembled the normal SC developmental progression. On the basis of the information from their conditional mouse models, Zhu et al. (2005) and Parada et al. (2005) hypothesize that inactivation of NF1 in SC precursors or in neural crest stem cells, but not in differentiated SCs, is required for neurofibroma formation. Our model system will allow us to test this hypothesis further in future experiments by implanting SC-like cells derived from mES cells along mouse nerve roots in both NF1 compromised mice and in their unaffected littermates to determine tumor status and tumor progression.

In vivo, there is a close relationship between neurons and SCs (Bunge et al., 1980; Lobsiger et al., 2002; Wood, 1976). Myelinating SC wrap around one or more individual large-diameter axons; nonmyelinating SCs insulate small-diameter neuron bundles that are imbedded in the cytoplasm and surrounded by plasma membrane (Lobsiger et al., 2002). SCs are unlikely to express myelin unless they are in the presence of neurons (Coman et al., 2005; Lobsiger et al., 2002). Genes that are upregulated later in SC development (PMP22) as well as mature SC genes expressed in myelinating (MBP) and nonmyelinating ( $\alpha 1 \beta$ 1integrin) SC were expressed at their highest levels in the cultures in our study only following exposure to SAG neurites, with the exception of DKOSC cells' MBP expression, which was decreased in the presence of SAGs. This could be due to overproliferation, aberrant SC/neurite interactions, or because the SAGs showed greater branching of neurites when grown in co-culture with DKOSC cells, which may result in the production of smaller diameter axons that are not myelinated. Two characteristics of neurofibromas include hypercellularity of SC and disengagement of SC from axons (Ling et al., 2005). In our study, although there was a consistent and close association between the D3SC and primary neurites of the SAG, and in some cases, SKOSC and DKOSC were also found associated with neurites, there were also large numbers of these neurofibromin compromised SC that remained unassociated with neurites and which grew in large clumps.

Consistent with NF1's function as an inactivator of the Ras pathway (Friedman et al., 1999; Kogut et al., 2007; Mattingly et al., 2006), DKOSC (NF1-/-) and SKOSC $(\mathrm{NF} 1+/-)$ cells showed significantly increased cell proliferation rates when compared to D3SC cells, and aberrant proliferative cells unassociated with SAG neurites in co-culture. When we inhibited this pathway using U0126, a Mek inhibitor (Favata et al., 1998; Kogut et al., 2007; Mattingly et al., 2006), we were able to return proliferation rates to WT levels. This indirectly indicates that the inability of neurofibromin-deficient cells to hydrolyze Ras effectively resulted in overproliferation. D3SC $(\mathrm{NF} 1+/+)$ cells, which are able to hydrolyze and inactivate Ras, were unaffected by U0126 addition. However, more direct tests of Ras pathway involvement are needed, including direct measurements of ERK1/2 phosphorylation (Adler et al., 2005), before we can do more than suggest a Ras involvement in these processes. 
To stimulate myelin expression and to determine axonal association, we examined the ability of the mESderived SC-like cells to interact with neurites of primary chick statoacoustic ganglia (SAG). The SAG innervates the inner ear and individual neurites are myelinated (Bianchi et al., 2005; Whitlon et al., 2006). It has been suggested that direct interaction with $\mathrm{SC}$ serves as a substrate for neuron attachment and growth, such as with SAGs (Whitlon et al., 2006). We found that neurite outgrowth from SAGs was increased in the presence of $\mathrm{mES}$ cells that had become SC-like, when compared to undifferentiated mES cells, mouse embryonic fibroblasts (not shown) or in media alone without an underlying cell layer. This was the case regardless of their NF1 genotype and NF1 expression level, although NF1 deficient cells exhibited more elaborate branching and smaller diameter neurites. The SAGs grown on SC-like cells supported extensive directional neurite outgrowth over the 5 -day culture period. SAG neurites that had begun to grow out from the side of the explant nearer the undifferentiated ES cells turned and extended towards the differentiated SC-like $\mathrm{mES}$ cells, suggesting either a chemoattractant or growth-promoting signal from the SC-like cells or a repulsive signal from the undifferentiated mES cells. We have recently found that these SClike cells make and upregulate two neurite outgrowth promoting cytokines, macrophage migratory inhibitory factor (MIF), and monocyte chemoattractant protein 1 (MCP1) (data not shown), which we have demonstrated is a powerful neurotrophic cytokine for SAG neurons (Bianchi et al., 2005). Ratner's group (Ling et al., 2005) also reported MCP1 to be upregulated in SC during tumor progression. SC-like mES cells express myelin protein and associate with axons in the presence of SAG neurites. SC and SC-like cells that expressed myelin clustered around the neurite processes. In the two NF1+/+ cell lines (D3 and SW10), myelin-positive cells clustered around the axons, but there was very little cell growth on the rest of the plate, again suggesting a possible chemoattractant component produced by the SC-like cells that could be MCP1 or MIF. We could not, however, document any evidence of SC-like cells wrapping around neurites in these cultures, as did others with primary SC and neurons in culture (Ratner et al., 1986). SC that wrap around neurons greater than $1 \mu \mathrm{M}$ diameter have been shown to myelinate, while SC associated with axons smaller than $1 \mu \mathrm{M}$ diameter are nonmyelinating. This could explain why MBP (myelinating SC) expression was higher in D3SC/SAG co-cultures and

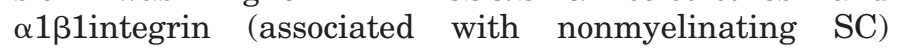
expression was higher in SKOSC/SAG and DKOSC/SAG co-cultures.

From these experiments we conclude that NF1 $(+/+$, $+/-$, or $-/-)$ mESC can differentiate in vitro into SClike cells that express appropriate SC genes in the expected order of expression for SC maturation. They support, and preferentially direct primary ganglion neurite outgrowth, and express myelin in cells near neurites. In vitro, $\mathrm{NF} 1+/+$ SC-like cells (D3SC) are more likely to express myelinating SC genes, and become qui- escent in the presence of SAG neurites at a rate similar to SW10 mouse SC. NF1+/- (SKOSC) and NF1-/- SClike cells (DKOSC) are more likely to stimulate greater neurite outgrowth and branching, express nonmyelinating genes, and slightly reduce proliferation in the presence of SAG neurites. Overproliferation of NF1 deficient (SKOSC) and null (DKOSC) SC-like cells can be reduced to $\mathrm{NF}+/+$ (D3SC) levels by the addition of a Mek inhibitor, indirectly indicating Ras pathway involvement. This paper is the first of which we are aware that shows that mES cells can be preferentially directed down the SC pathway. Since all three mES cell lines, regardless of their NF1 status, can respond by differentiating into SC-like cells, these cells can be used in further studies of the function of $\mathrm{SC}$ with varying levels of neurofibromin in tumor formation and growth.

\section{ACKNOWLEDGMENTS}

Thanks to Dr. Tyler Jacks (MIT) for the NF1 ES cell lines. Thanks to Naweah P. Attia, Luming Feng and Patricia Lenderman for excellent technical assistance. Dr. David Molea and James Iannuzzi also helped with SAG culture. Thanks to Dr. Yuan Zhu for a critical reading of this manuscript.

\section{REFERENCES}

Adler V, Qu Y, Smith SJ, Izotova L, Pestka S, Kung H-F, Lin M, Friedman FK, Chie L, Chung D, Boutjdir M, Pincus MR. 2005. Functional interactions of Raf and MEK with Jun-N-terminal kinase (JNK) result in a positive feedback loop on the oncogenic Ras signaling pathway. Biochemistry 44:10784-10795.

Bianchi LM, Daruwalla Z, Roth TM, Attia NP, Lukacs NW, Richards AL, White IO, Allen SJ, Barald KF. 2005. Immortalized mouse inner ear cell lines demonstrate a role for chemokines in promoting the growth of developing statoacoustic ganglion neurons. J Assoc Res Otolaryngol 6:355-367.

Bunge MB, Williams AK, Wood PM. 1982. Neuron-Schwann cell interaction in basal lamina formation. Dev Biol 92:449-460.

Bunge MB, Williams AK, Wood PM, Uitto J, Jeffrey JJ. 1980. Comparison of nerve cell and nerve cell plus Schwann cell cultures, with particular emphasis on basal lamina and collagen formation. J Cell Biol 84:184-202.

Cichowski K, Jacks T. 2001. NF1 tumor suppressor gene function: Narrowing the GAP. Cell 104:593-604.

Coman I, Barbin G, Charles P, Zalc B, Lubetzki C. 2005. Axonal signals in central nervous system myelination, demyelination and remyelination. J Neurol Sci 233:67-71.

Daston MM, Ratner N. 1992. Neurofibromin, a predominantly neuronal GTPase activating protein in the adult, is ubiquitously expressed during development. Dev Dyn 195:216-226.

Daston MM, Scrable H, Nordlund ML, Sturbaum AK, Nissen LM, Ratner N. 1992. The protein product of the Neurofibromatosis Type 1 gene is expressed at highest abundance in neurons, Schwann cells, and oligodendrocytes. Neuron 8:415-428.

Doetschman TC, Eistetter H, Katz M, Schmidt W, Kemler R. 1985. The in vitro development of blastocyst-derived embryonic stem cell lines: Formation of visceral yolk sac, blood islands and myocardium. J Emb Exp Morph 87:27-45.

Dorsky RI, Moon RT, Raible DW. 2000. Environmental signals and cell fate specification in premigratory neural crest. Bio Essays 22:708-716.

Favata MF, Horiuchi KY, Manos EJ, Daulerio AJ, Stradley DA, Feeser WS, Van Dyk DE, Pitts WJ, Earl RA, Hobbs F, Copeland RA, Magolda RL, Scherle PA, Trzaskos JM. 1998. Identification of a novel inhibitor of mitogen-activated protein kinase kinase. J Biol Chem 273:18623-18632.

Friedman JM, Gutmann DH, MacCollin M, Riccardi VM. 1999. Neurofibromatosis: Phenotype, natural history, and pathogenesis. Baltimore: Johns Hopkins University Press. 381 p. 
Gutmann DH, Wu YL, Hedrick NM, Zhu Y, Guha A, Parada LF. 2001 Heterozygosity for the neurofibromatosis 1 (NF1) tumor suppressor results in abnormalities in cell attachment, spreading and motility in astrocytes. Hum Mol Genet 10:3009-3016.

Harrisingh MC, Lloyd AC. 2004. Ras/Raf/ERK signalling and NF1. Cell Cycle 3:1255-1258.

Jacks T, Shih TS, Schmitt EM, Bronson RT, Bernards A, Weinberg RA. 1994. Tumour predisposition in mice heterozygous for a targeted mutation in Nf1. Nat Genet 7:353-361.

Jessen KR, Mirsky R. 1991. Schwann cell precursors and their development. Glia 4:185-194.

Kalcheim C, Burstyn-Cohen T. 2005. Early stages of neural crest ontogeny: Formation and regulation of cell delamination. Int $J$ Dev Biol 49:105-116.

Kogut MH, Genovese KJ, He H. 2007. Flagellin and lipopolysaccharide stimulate the MEK-ERK signaling pathway in chicken heterophils through differential activation of the small GTPases, Ras and Rap1. Mol Immunol 44:1729-1736.

Le Douarin NM, Creuzet S, Couly G, Dupin E. 2004. Neural crest cell plasticity and its limits. Development 131:4637-4650.

Lee PR, Cohen JE, Tendi EA, Farrer R, GH DEV, Becker KG, Fields RD. 2004. Transcriptional profiling in an MPNST-derived cell line and normal human Schwann cells. Neuron Glia Biol 1:135-147.

Ling BC, Wu J, Miller SJ, Monk KR, Shamekh R, Rizvi TA, DecourtenMyers G, Vogel KS, DeClue JE, Ratner N. 2005. Role for the epidermal growth factor receptor in neurofibromatosis-related peripheral nerve tumorigenesis. Cancer Cell 7:65-75.

Lisak RP, Bealmear B. 1991. Antibodies to interleukin-1 inhibit cytokine-induced proliferation of neonatal rat Schwann cells in vitro. J Neuroimmunol 31:123-132.

Lobsiger CS, Taylor V, Suter U. 2002. The early life of a Schwann cell. Biol Chem 383:245-253.

Mattingly RR, Kraniak JM, Dilworth JT, Mathieu P, Bealmear B, Nowak JE, Benjamins JA, Tainsky MA, Reiners JJ Jr. 2006. The mitogen-activated protein kinase/extracellular signal-regulated kinase kinase inhibitor PD184352 (CI-1040) selectively induces apoptosis in malignant schwannoma cell lines. J Pharmacol Exp Ther 316:456-465.

Miller SJ, Rangwala F, Williams J, Ackerman P, Kong S, Jegga AG, Kaiser S, Aronow BJ, Frahm S, Kluwe L, Mautner V, Upadhyaya M,
Muir D, Wallace M, Hagen J, Quelle DE, Watson MA, Perry A, Guttman DH, Ratner N. 2006. Large-scale molecular comparison of human Schwann Cells to malignant peripheral nerve sheath tumor cell lines and tissues. Cancer Res 66:2584-2591.

Parada LF, Kwon CH, Zhu Y. 2005. Modeling neurofibromatosis type 1 tumors in the mouse for therapeutic intervention. Cold Spring Harb Symp Quant Biol 70:173-176.

Ratner N, Elbein A, Bunge MB, Porter S, Bunge RP, Glaser L. 1986. Specific asparagine-linked oligosaccharides are not required for certain neuron-neuron and neuron-Schwann cell interactions. J Cell Biol 103:159-170.

Rutkowski JL, Wu K, Gutmann DH, Boyer PJ, Legius E. 2000. Genetic and cellular defects contributing to benign tumor formation in neurofibromatosis type 1. Hum Mol Genet 9:1059-1066.

Sawada S, Florell S, Purandare SM, Ota M, Stephens K, Viskochil D. 1996. Identification of NF1 mutations in both alleles of a dermal neurofibroma. Nat Genet 14:110-112.

Serra E, Rosenbaum T, Winner U, Aledo R, Ars E, Estivill X, Lenard HG, Lazaro C. 2000. Schwann cells harbor the somatic NF1 mutation in neurofibromas: Evidence of two different Schwann cell subpopulations. Hum Mol Genet 9:3055-3064.

Sieber-Blum M, Grim M, Hu YF, Szeder V. 2004. Pluripotent neural crest stem cells in the adult hair follicle. Dev Dyn 231:258-269.

Vogel KS, Brannan CI, Jenkins NA, Copeland NG, Parada LF. 1995 Loss of neurofibromin results in neurotrophin-independent survival of embryonic sensory and sympathetic neurons. Cell 82:733-742.

Whitlon DS, Ketels KV, Coulson MT, Williams T, Grover M, Edpao W, Richter CP. 2006. Survival and morphology of auditory neurons in dissociated cultures of newborn mouse spiral ganglion. Neuroscience 138:653-662.

Wood PM. 1976. Separation of functional Schwann cells and neurons from normal peripheral nerve tissue. Brain Res 115:361-375.

Zhu Y, Ghosh P, Charnay P, Burns DK, Parada LF. 2002. Neurofibromas in NF1: Schwann cell origin and role of tumor environment. Science 296:920-922.

Zhu Y, Guignard F, Zhao D, Liu L, Burns DK, Mason RP, Messing A Parada LF. 2005. Early inactivation of p53 tumor suppressor gene cooperating with NF1 loss induces malignant astrocytoma. Cancer Cell 8:119-130 